Article

\title{
Effect of a Chitosan-Based Biodegradable Middle Meatal Dressing after Endoscopic Sinus Surgery: A Prospective Randomized Comparative Study
}

\author{
Kevin Hsu, Matthew Ericksen ${ }^{\dagger}$ and Peter Catalano ${ }^{\dagger}, *$ \\ Department of Otolaryngology, St Elizabeth's Medical Center, 736 Cambridge Street, \\ SMC-8 Brighton, MA 02135, USA; E-Mails: kevin.hsu@steward.org (K.H.); \\ matthew.ericksen@steward.org (M.E.) \\ $\dagger$ These authors contributed equally to this work. \\ * Author to whom correspondence should be addressed; E-Mail: Peter.Catalano@steward.org; \\ Tel.: +617-779-6440; Fax: +617-779-8483.
}

Academic Editor: Claudina A. Pérez Novo

Received: 10 October 2015 / Accepted: 7 November 2015 / Published: 25 November 2015

\begin{abstract}
Introduction: The use of biomaterials to improve wound healing after endoscopic sinus surgery (ESS) is not new. Many types of resorbable and non-resorbable materials have been tried as a middle meatal (MM) dressing, spacer, or stent to prevent lateralization of the middle turbinate, formation of synechia, granulation tissue, adhesions and scarring. The FDA has recently approved Chitosan-based nasal dressing/spacers which have optimal wound healing characteristics, including hemostatic and bacteriostatic properties. Herein, we compare a new chitosan-based biomaterial to a popular fully synthetic resorbable dressing in patients undergoing ESS. Materials and Methods: A prospective randomized controlled study was performed comparing a new Chitosan-based bioresorbable nasal dressing (Posi-Sep X) against a previously studied and well known fully synthetic polyurethane-based control (Nasopore). Post-operative outcome metrics included the degree of crusting, amount of retained implant, patient comfort, wound healing, epistaxis, and post-operative infection at two weeks. Results: Thirty-five patients were enrolled and a total seventy implants were placed $(n=70)$ at the completion of ESS. The results show a statistically significant difference between the Chitosan-based product and the control with respect to wound healing, degree of crusting, and resorption profile. In addition, the Chitosan-based dressing had a markedly lower requirement for post-operative debridement, and a lower incidence of
\end{abstract}


epistaxis and infection, which corresponds to superior patient comfort. Conclusion: Our study is consistent with the biomaterials literature regarding the potential advantages of Chitosan-based MM dressings after ESS regarding improved wound healing, biocompatibility, and patient comfort.

Keywords: chitosan; biodegradable; middle meatal dressing; middle meatal spacer; endoscopic sinus surgery; NasoPore ${ }^{\mathrm{TM}}$; Posi-Sep $\mathrm{X}^{\mathrm{TM}}$; sinus stent

\section{Introduction}

Since the late 1980's, endoscopic sinus surgery (ESS) has become the most widely adopted technique and the standard of care for sinus surgery [1]. The transition from the Caldwell-Luc and other external approaches to functional and minimally invasive sinus techniques has presented new challenges, with the success of ESS heavily influenced by wound healing. Prior to MM dressings, frequent post-operative debridements in the operating room or clinic were required to assist and modulate wound healing. With the modern emphasis on further tissue preservation within the MM, and thus less dependence on frequent post-operative debridements, the emphasis and opportunity for better wound healing has shifted to the use of inert resorbable biomaterials as MM dressings after ESS [2].

There have been numerous studies highlighting the consequences of abnormal wound healing including synechia, scarring, middle meatal obstruction, lateralization of the middle turbinate, recurrent infection, epistaxis, crusting, granuloma formation, and persistent inflammation. All of these are associated with poor surgical outcomes $[1,2]$.

In addition to wound healing, patient comfort is an increasingly important consideration in the management of patients after ESS, and it is often dictated by the type and extent of surgery, the type and amount of nasal dressings used, the amount of MM crusting requiring post-operative debridement, and post-operative complications that required additional procedures or medications.

The trend towards accelerated wound healing while maximizing patient comfort has been associated with the use of biocompatible MM dressings, with or without the inclusion of topical medications. Various types of resorbable MM dressings have demonstrated improvements in wound healing compared to non-resorbable dressings such as Vaseline gauze, silicon-stents, Merocel sponges, and finger cots stuffed with cotton. There is currently no standard or recommendation regarding the use or type of MM dressing that best achieves its desired purpose, and only a limited number of studies that offer a direct head to head comparison on the efficacy of different materials [3].

Various types of resorbable MM dressings have been developed and marketed in the last 15 years ranging from Carboxy-methyl-Cellulose (CMC) foam, collagen-based gel foam, hyaluronic acid-based films and foams, to polyurethane sponges. As part of this effort, multiple types of materials with different bioreactivity, biodegradability, and degradation kinectics have been investigated for their potential wound healing benefits. Thus far, a fully synthetic polyurethane sponge (Nasopore), has shown one of the most favorable biocompatibility and bioresorption profiles, with excellent wound healing characteristics $[1,3-5]$. However, many surgeons find the degradation time of polyurethane to be too long ( $>7$ days), and the size and stiffness of the foam affects ease of use for the surgeon. 
A bioresorbable Chitosan-based nasal dressing recently approved by the FDA has drawn interest due to Chitosan's hydrophilic, antibacterial, and hemostatic properties, as well as its ability to minimize postop adhesions, synechia, and crusting when in contact with mucosal membranes. Both of these materials also possess the ability to act as a carrier for the targeted delivery of topical medications, such as antibiotics and/or steroids, which have been shown to further enhance wound healing [2,6,7].

Chitosan is a polysaccharide molecule derived from de-acylation of chitin from the exoskeletons of crustaceans. Multiple biomedical applications and research in the military have shown the inherent antibacterial and hemostatic properties of Chitosan, which improves wound healing for advanced biomedical use $[6,7]$.

This study investigates and compares the wound healing and hemostatic characteristics, and patient comfort factors of two bioresorbable MM dressings, a Chitosan-based polymer (PosiSep X) and a well-known fully synthetic polyurethane foam sponge (NasoPore) in patients recovering from ESS [3].

\section{Experimental Section}

All materials used are FDA approved for nasal cavity dressing application following ESS. Institutional review board approval was obtained for the study. The study design is a prospective randomized controlled cohort with 35 patients enrolled and selected for medically refractory bilateral ESS with or without septoplasty, as indicated by AAO-HNS chronic rhinosinusitis diagnostic criteria guidelines (2015) [4].

Seventy total implants $(n=70)$ were randomized into two arms. All dressings were placed in the MM at the completion of ESS. The control group received the polyurethane sponge soaked in bacitracin solution, while the study group received the Chitosan-based product with bacitracin solution [8]. Patients were blinded as to which MM dressing they received.

Patients were endoscopically assessed for the first time at their two week post-op visit for the following metrics: degree of crusting (rated from none (0) mild (1), moderate (2), severe (3)), amount of retained implant (none, up to $50 \%$, or $>50 \%$ ), epistaxis, infection, allergic reactions, and granulation formation.

All patients began nasal saline irrigations on post-op day 1 and continued them twice daily for post-operative care. No one received oral steroids, oral antibiotics, antihistamines or topical nasal steroids post-operatively. We have previously shown topical targeted delivery of bacitracin in a polyurethane sponge (off-label use) to be as effective as oral antibiotics in the prevention of infection following ESS $[2,8]$.

Because we understand that there is no accurate objective means to determine the exact amount of crusting or retained material in the post-operative MM, we have tried to simplify the metrics accordingly. Thus, amount of retained material was either none, up to $50 \%$ or greater than $50 \%$ of the original amount, and crusting was graded as mild, moderate or severe. Two surgeons not involved in the surgery had to agree on the post-operative endoscopic scoring before assigning a value to any given patient. The patients did not serve as their own control, with one side receiving polyurethane and the other chitosan, because this would eliminate the ability to assess subjective outcomes. Thus, two separate groups were used which were randomized and blinded to the product they received.

Of the 16 patients in the control group, there were 9 male and 7 females. Age ranged from 9 to 77 years. All 16 underwent septoplasty, bilateral anterior ethmoidectomy, uncinectomy, and 
radiofrequency reduction of the inferior turbinates. In the Chitosan group, there were 14 males and 5 females, with ages ranging from 9 to 71 years. All 19 patients underwent septoplasty, bilateral anterior ethmoidectomy, uncinectomy, and radio-frequency reduction of the inferior turbinates. There were no differences between groups with respect to gender, age, or extent and type of surgery (see Table 1).

\section{Results and Discussion}

\subsection{Results}

Thirty-five patients enrolled with seventy MM dressings placed (Total $N=70$, see Table 1 ). Sixteen patients ( 32 dressings) comprised the control group and 19 patients ( 38 dressings) received the Chitosan product. Each patient was examined at two weeks post-ESS, and scored according to the degree of crusting and percentage of retained material as seen on endoscopy. Also recorded was the presence of any granulomas, synechia, and the incidence of adverse events or complications such as infections, epistaxis, or allergic reactions.

Table 1. Demographics and Patient Characteristics.

\begin{tabular}{cc}
\hline Demographics & \\
\hline Number of Patients & 35 \\
Number of Implants (n) & 70 \\
Average Age & 39 \\
std dev & 21 \\
min age & 9 \\
max age & 77 \\
\% Female & 34 \\
\% Male & 66 \\
\% CRSwNP & 11 \\
\% CRSsNP & 89 \\
\% Primary ESS & 77 \\
\% Revision ESS & 23 \\
\% AR & 46 \\
\% DM & 3 \\
\% Coagulopathic & 3 \\
\hline
\end{tabular}

The average degree of crusting (0-3) for the polyurethane/control group was 1.23 (std dev 0.81), indicating mild to moderate crusting. For the Chitosan group, the average degree of crusting was 0.53 (std dev 0.51), indicating mostly an absence of, or only mild crusting (Table 2 and Figure 1). Paired $t$-tests, used to determine the significance of the difference between groups for the average degree of crusting showed a $p$-value $=0.000033$, which was statistically significant (Figure 2). 
Table 2. Tracking of each dressing categorized by the degree of crusting.

\begin{tabular}{ccc}
\hline Crusting & Control & Chitosan \\
\hline None (0) & 5 & 18 \\
Mild (1) & 15 & 20 \\
Moderate (2) & 10 & 0 \\
Severe (3) & 2 & 0 \\
Total Number & 32 & 38 \\
\hline
\end{tabular}

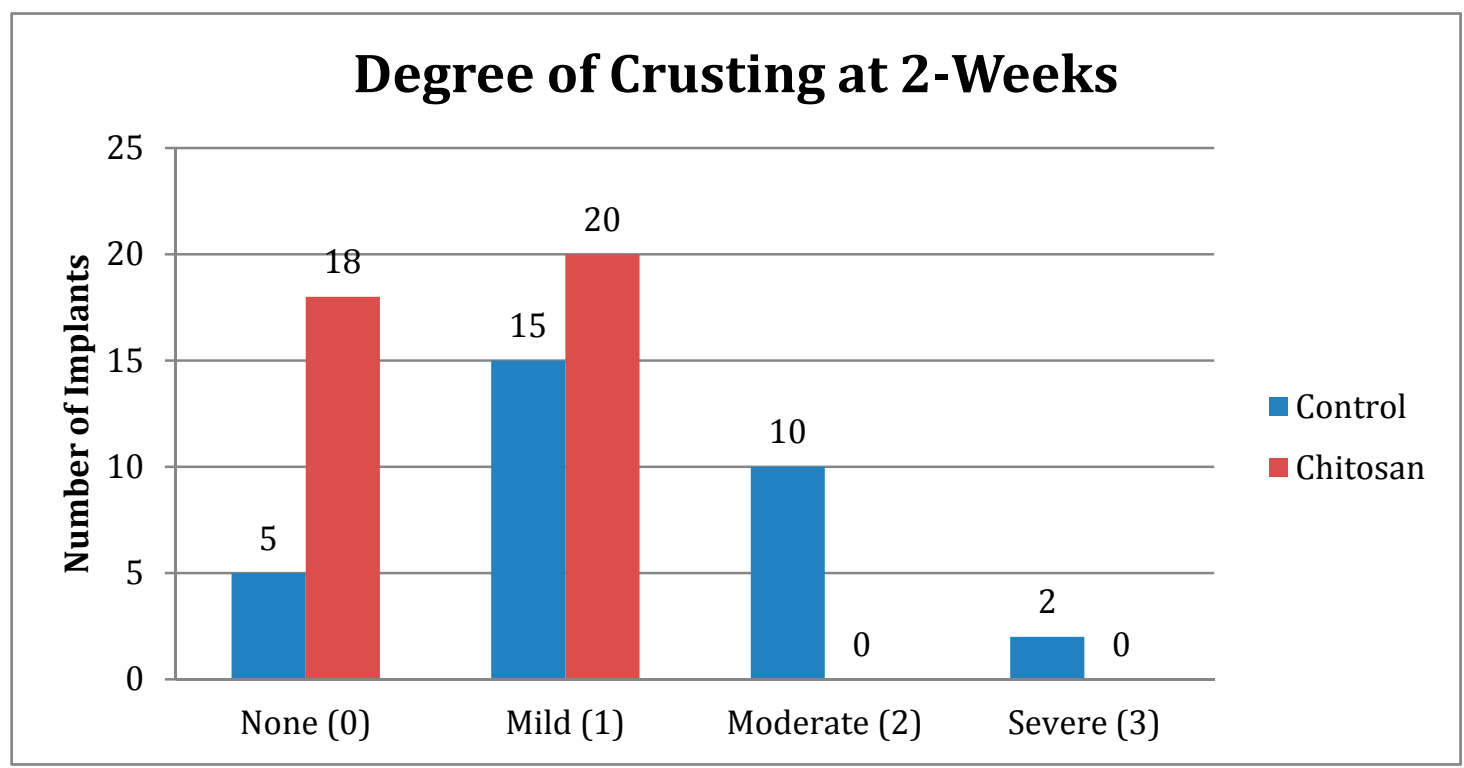

Figure 1. Degree of crusting at 2-Weeks for the control vs. Chitosan.

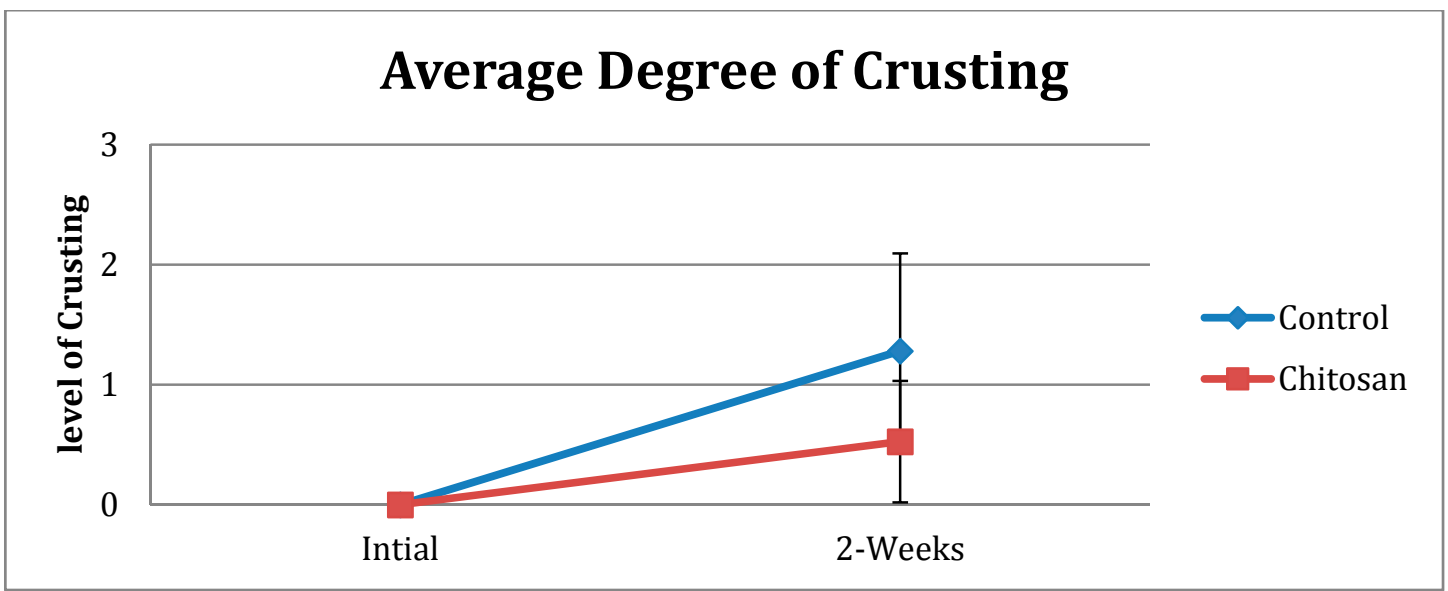

Figure 2. Average Degree of crusting $($ None $=0$, Mild $=1$, Moderate $=2$, Severe $=3$ ) for control vs. Chitosan.

The average percentage of retained implant at two weeks for the control group was $36 \%$ (std dev 36.44), whereas for the Chitosan group, the percentage of retained material was $<1 \%$ (Std dev 4.06). The latter result means most of the Chitosan material had dissolved within two weeks (Table 3 and Figure 3). Paired t-tests used to determine the significance of the difference in the average percentage of retained material between groups showed a $p$-value $=0.000006$, which is highly significant (Figure 4). 
Table 3. Tracking of each material categorized by the $\%$ retained.

\begin{tabular}{ccc}
\hline Retained Dressing & Control & Chitosan \\
\hline None & 15 & 37 \\
Up to $50 \%$ & 7 & 1 \\
More than $50 \%$ & 10 & 0 \\
Total Number & 32 & 38 \\
\hline
\end{tabular}

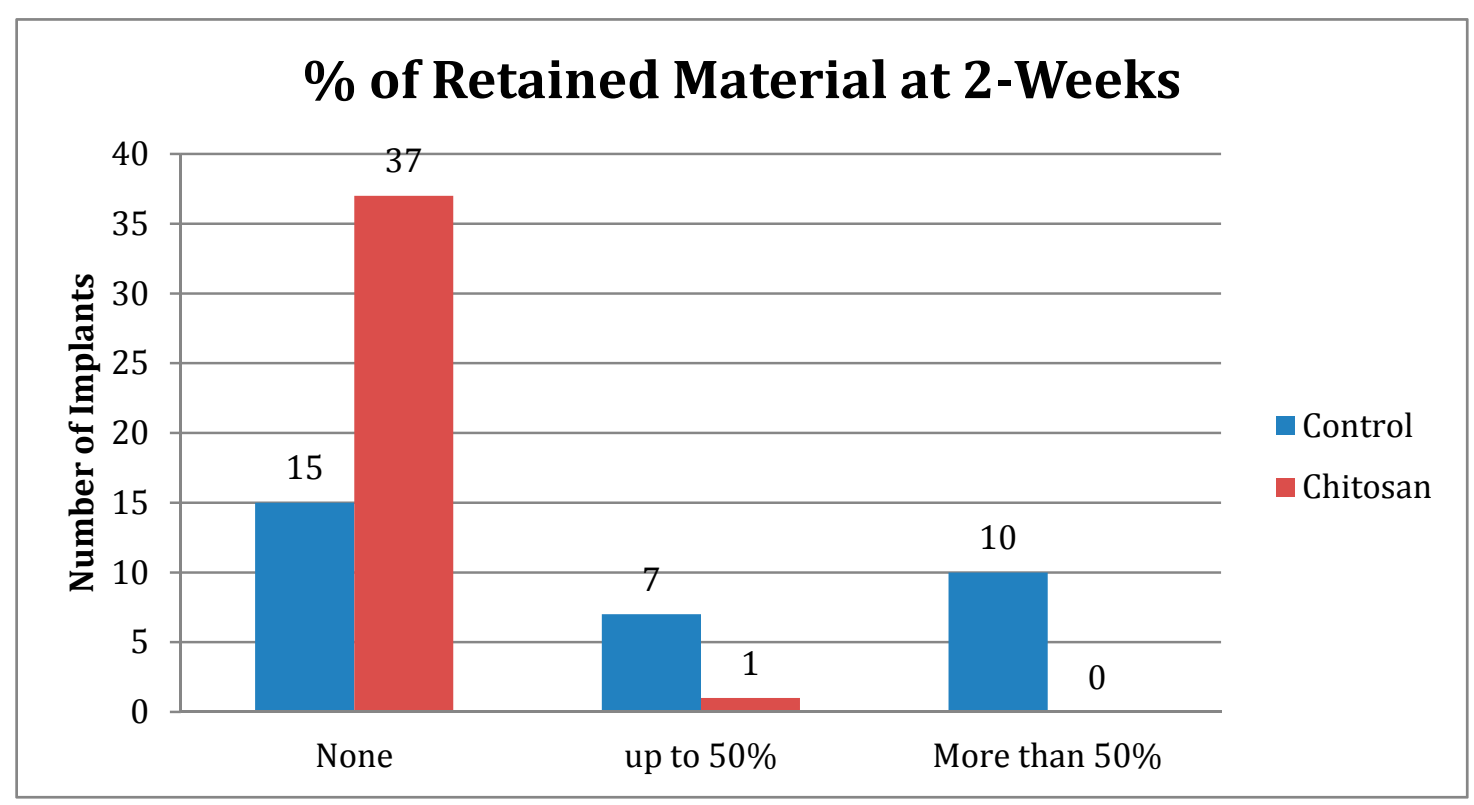

Figure 3. \% of Retained Material at 2-Weeks for Control vs. Chitosan.

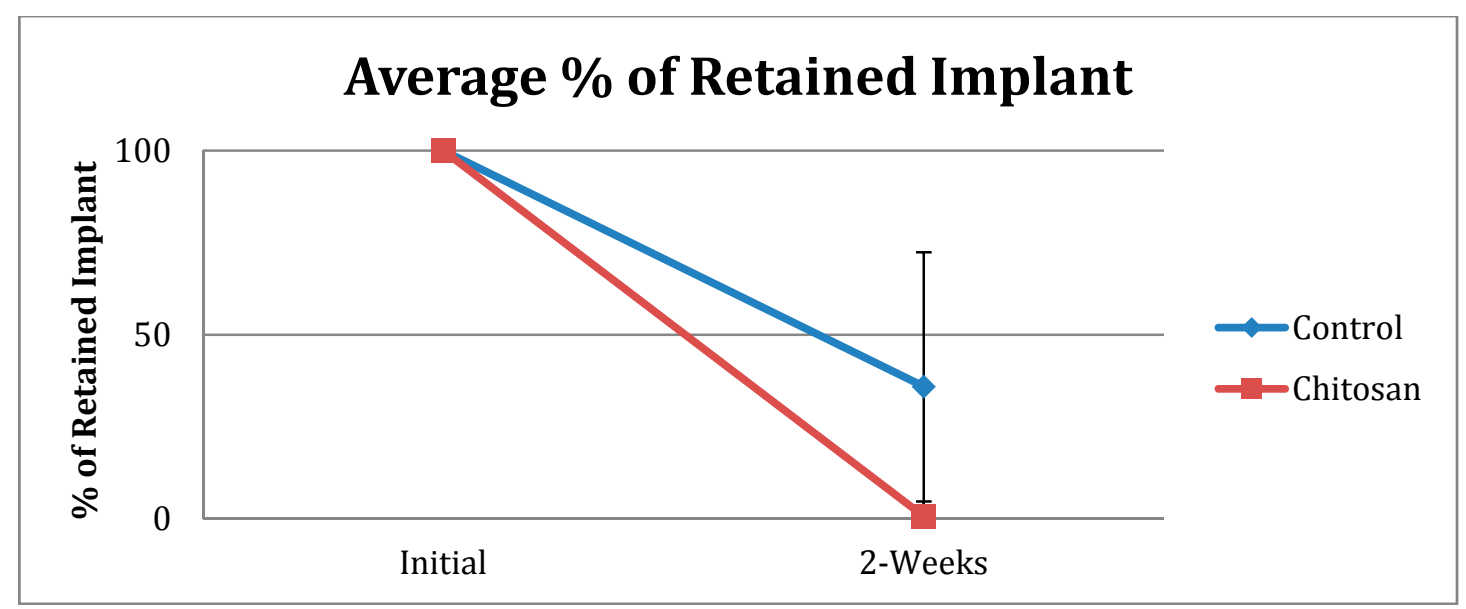

Figure 4. Average \% of Retained Implant for Control vs. Chitosan.

A correlation graph plotting the degree of crusting against the percentage of retained material for the control group showed that more retained material within the MM was associated with more crusting; this phenomenon was not observed in the Chitosan group (Figure 5). 


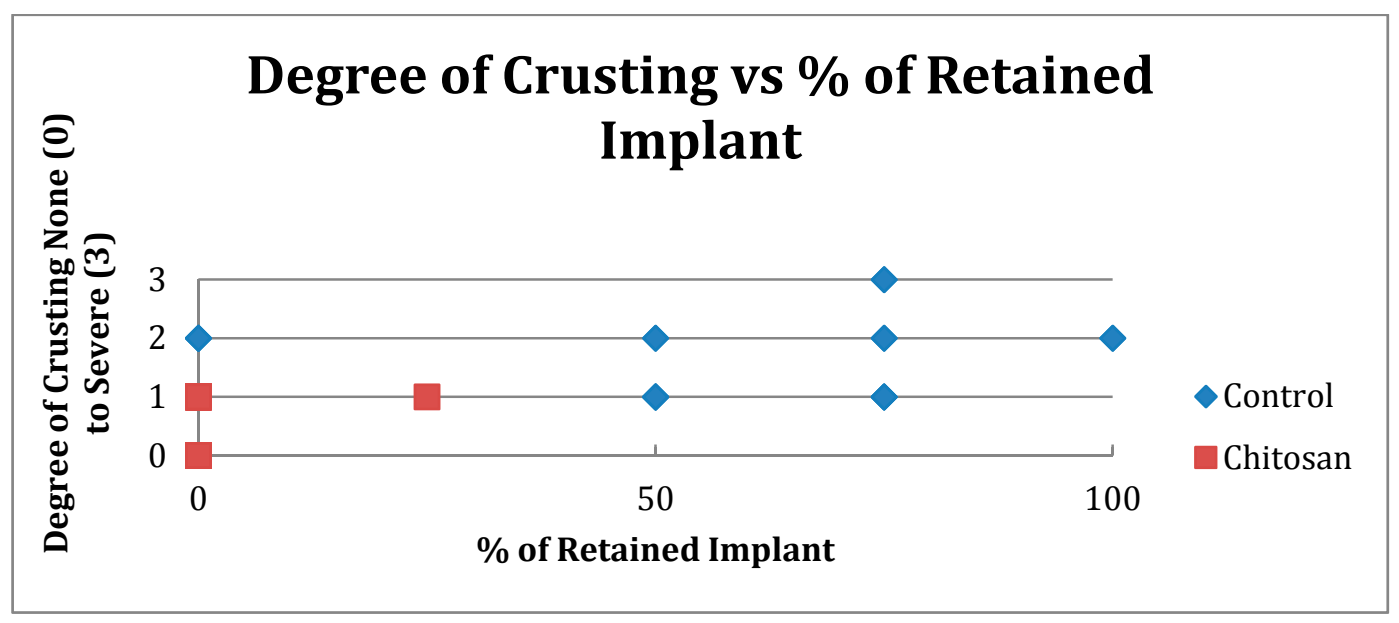

Figure 5. Degree of crusting (Scale 0-3) vs. \% of Retained Material (None, Up to 50\%, More than 50\%).

Within the control group, two patients with a normal pre-operative coagulation profile had post-operative epistaxis (13\%), and one patient developed a post-operative infection (6\%) requiring oral antibiotics and additional endoscopic debridement. No complications were reported in the Chitosan group.

None of the patients in either group exhibited adverse synechia, granuloma formation, or allergic reaction during the follow-up period.

Finally, patient comfort, measured by the necessity for and extent of post-operative endoscopic debridement, showed that $56 \%$ of the control group required endoscopic debridement compared to only $11 \%$ for the Chitosan group (See Table 4).

Table 4. Requirement for Post-operative Debridement for the Control vs. Chitosan group.

\begin{tabular}{cccc}
\hline \multicolumn{4}{c}{ Requirement for Post-Operative Endoscopic Debridement } \\
\hline & Occurrence & Total Patient & \% Incidence \\
\hline Control & 9 & 16 & 56 \\
Chitosan & 2 & 19 & 11 \\
\hline
\end{tabular}

\subsection{Discussion}

Over the past 5 years, polyurethane foam nasal dressings have become very popular and were our preferred choice for nasal dressings after ESS [3]. This product consistently outperformed other bioresorbable MM dressings including collagen-based gelatin, hyaluronic acid, and carboxy-methyl cellulose [1]. In a previous study comparing hyaluronic acid based merogel to polyurethane foam, the hyaluronic acid dressing formed inclusion bodies within the mucosal surface of the surgical wound. This is a typical foreign body reaction based on histologic analysis [4]. Yan et al. showed that other types of materials caused more crusting and had a higher percentage of retained implant on gross endoscopic examination at two weeks after ESS [1]. Both microscopic and macroscopic foreign body reactions can delay proper wound healing, which often leads to additional procedural or medical interventions. In general, a MM dressing with poor biocompatibility reduces patient comfort and satisfaction after ESS $[1,2,4,9]$. There have also been studies using MM dressings containing fibrin and/or thrombin that 
demonstrated excellent hemostatic properties. However, these studies often found a high incidence of synechia and granuloma formations with a delayed bioresorption profile making them less favorable and dependent on additional post-ESS debridements [10,11].

In the last five years, numerous biomaterial research studies had concluded that Chitosan is a versatile material with enhanced hemostatic and antimicrobial properties, especially for wound healing $[12,13]$. In addition, Chitosan materials can also be fabricated with various degrees of porosity, de-acylation, and incorporation of medication for targeted delivery that further improves the biocompatibility, bioresorption, and desired drug delivery kinetics without interfering with wound healing [14,15].

In this study, topical bacitracin solution, as shown by Wijewickrama, et al., was used in both the polyurethane and Chitosan dressings at the completion of ESS [16]. This off-label protocol has become our norm and has allowed the elimination of routine post-operative oral antibiotics after ESS which is extremely convenient and cost-effective for patients. It is unknown whether the topical antibiotics have any impact on wound healing or crusting, but whatever their influence, it should not be different between the two groups. In Wijewickrama's study, Nasopore was the resorbable dressing used in over 350 patients after ESS, and there was no reported interaction between Nasopore and Bacitracin in any patient. Similarly, based on our own personal experience, we have not seen any adverse effect from using Bacitracin solution in our resorbable dressings in over 500 cases of ESS.

This study aimed to directly compare the new Chitosan-based nasal dressing against polyurethane foam, our current MM dressing of choice, using the same operative and post-operative protocol to control all possible confounding factors. The follow-up period is purposely limited to 2 weeks because this is the time when retained material in the MM can cause problems with wound healing, patient comfort, and the need for repeated debridements. Previous reports on the polyurethane sponge dressing have shown it to be biologically inert and well tolerated in the MM during the first 90 days after ESS $[3,9,17,18]$. The chitosan dressing is reported to dissolve away sooner than polyurethane and has also been shown to be biologically inert by its chemical nature [12,13]. Therefore, it did into seem necessary to extend the follow-up period beyond 2 weeks because we were most interested in the short-term effect of each dressing.

The results of this prospective randomized controlled study demonstrated that the Chitosan-based dressing proved superior in material resorption and degree of crusting than the control group $(p<0.000001)$.

To answer the question of whether the degree of crusting was directly related to the amount of retained MM material, the correlation graph was generated (Figure 5). The graph shows a directly proportional relationship only in the control group; the same phenomenon was not observed in the Chitosan group.

Interestingly, the two patients who had post-operative epistaxis were in the control group, despite having a normal coagulation profile and similar extent of surgery to the rest of the group. However, there were 2 patients with a known coagulopathy in the study; one in the control group had factor VIII deficiency and the other, in the Chitosan group, was on Aspirin 325mg daily. Neither of them developed post-operative epistaxis.

No one in the study developed an allergic reaction to either biomaterial, and there was no granulation tissue or synechia seen at their two-week follow-up visit. While this time frame may be considered too short to develop either of these problems, the fact that any residual material was cleared from the MM during the first follow-up visit, and patients continued to irrigate with normal saline twice daily for 
another 2 weeks, make it very unlikely for either of these problems to develop at a later date from the biomaterial itself.

We have never used a suture to help medialize the MT during ESS. In our experience, the biomaterial placed in the MM during ESS usually prevents medialization, albeit not in all cases. If lateralization does occur, it is usually clinically insignificant and does not obstruct the maxillary sinus ostia. Our other concern is the risk of developing a contact point headache from this technique, however, we have little evidence to support this concern.

Thus, by direct comparison, our study shows that the Chitosan-based bioresorbable MM dressing had significantly better wound healing characteristics and enhanced quality measures leading to improved patient comfort.

\section{Conclusions}

A Chitosan-based biodegradable MM dressing offers a statistically significant advantage over a polyurethane-based biodegradable polymer with respect to wound healing characteristics measured by degree of crusting, amount of retained implant, epistaxis, and infection in patients undergoing ESS. Furthermore, the Chitosan dressing was also superior based on the decreased requirement for, and extent of, post-operative endoscopic debridement, and a lower complication rate as measured by the need for additional corrective procedures and/or medical intervention. Our study is consistent with the current scientific literature regarding Chitosan-based biomaterials that offer enhanced biocompatibility and are hydrophilic, bacteriostatic, and hemostatic.

\section{Acknowledgments}

Hussam Tallab MD contribution to the project's data collection.

\section{Author Contributions}

All authors contributed to the work of this paper. K.H. is the primary author of this paper with writing, research, editing, data analysis, and data collection. M.E. is the secondary author of this paper with editing, and data collection. P.C. is the principle investigator with research design, editing, and data analysis. All authors discussed, edited, and approved the final version.

\section{Conflicts of Interest}

The authors declare no conflict of interest.

\section{References}

1. Yan, M.; Zheng, D.; Li, Y.; Zheng, Q.; Chen, J.; Yang, B. Biodegradable nasal Packings for Endoscopic Sinus Surgery: A Systematic Review and Meta-Analysis. PLoS One 2014, 9, e115458. doi:10.1371/journal.pone.0115458.

2. Cote, D.W.; Wright, E.D. Triamcinolone-Impregnated Nasal Dressing Following Endoscopic Sinus Surgery: A Randomized, Double-Blind, Placebo-Controlled Study. Laryngoscope 2010, 120, 1269-1273. 
3. Catalano, P.J.; Payne, S.; Thong, M. Clinical Evaluation of a Fully Synthetic Middle Meatal Stent for Safety and Tolerability. Otolaryngol.-Head Neck Surg. 2011, 144, 452-456.

4. Catalano, P.J.; Roffman, E. Evaluation of middle meatal stenting after minimally invasive sinus techniques (MIST). Otolaryngol.-Head Neck Surg. 2003, 128, 875-881.

5. Weitzel, E.K.; Wormald, P.J. A scientific review of middle meatal packing/stents. Am. J. Rhinol. 2008, 22, 302-307.

6. Kheirabadi, B.S.; Acheson, E.M.; Deguzman, R.; Sondeen, J.L.; Ryan, K.L.; Delgado, A.; Dick, E.J., Jr.; Holcomb, J.B. Hemostatic efficacy of two advanced dressings in an aortic hemorrhage model in Swine. J. Trauma. 2005, 59, 25-34.

7. Pusateri, A.E.; McCarthy, S.J.; Gregory, K.W.; Harris, R.A.; Cardenas, L.; McManus, A.T.; Goodwin, C.W. Effect of a Chitosan-based hemostatic dressing on blood loss and survival in a model of severe venous hemorrhage and hepatic injury in swine. J. Trauma 2003, 54, 177-182.

8. Rosenfeld, R.M.; Piccirillo, J.F.; Chandrasekhar, S.S.; Brook, I.; Kumar, K.A.; Kramper, M.; Orlandi, R.R.; Palmer, J.N.; Patel, Z.M.; Peters, A.; et al. Clinical Practice Guideline (Update): Adult Sinusitis. Otolaryngol.-Head Neck Surg. 2015, 152, s1-s39.

9. Kastl, K.G.; Reichert, M.; Scheithauer, M.O.; Sommer, F.; Kisser, U.; Braun, T.; Havel, M.; Leunig, A. Patient comfort following FESS and NasoPore Packing, A double blind, prospective, randomized trial. Int. J. Rhinol. 2014, 52, 60-65.

10. Chandra, R.K.; Conley, D.; Kern, R. The effect of FloSeal on mucosal healing after endoscopic sinus surgery: A Comparison with Thrombin-Soaked Gelatin Foam. Am. J. Rhinol. 2003, 17, 51-55.

11. Shashoua, A.R.; Gill, D.; Barajas, R.; Dini, M.; August, C.; Kirschenbaum, G.L.; Escuardo, L. Caseating granulomata caused by hemostatic agent posing as metastatic leiomyosarcoma. J. Soc. Laparoendsc. Surg. 2009, 13, 226-228.

12. Ong, S.Y.; Wu, J.; Moochhala, S.M.; Tang, M.H.; Lu, J. Development of a chitosan-based wound dressing with improved hemostatic and antimicrobial properties. Biomaterials 2008, 29, 4323-4332.

13. Huang, X.; Sun, Y.F.; Nie, J.; Lu, W.; Yang, L.; Zhang, Z.; Yin, H.; Wang, Z.; Hu, Q. Using absorbable chitosan hemostatic sponges as a promising surgical dressing. Int. J. Biol. Macromol. 2015, 75, 322-329.

14. Wijewickrama, R.; Catalano, P.; Gupta, R.; Willen, S.; More, Y.; Jonnalagadda, S.; Warman, M. Efficacy of targeted middle metal antibiotics and endoscopic sinus surgery. Am. J. Rhinol. Allergy 2013, 27, 329-332.

15. Jayakumar, R.; Prabaharan, M.; Kumar, P.T.S.; Nair, S.V.; Tamura, H. Biomaterials based on chitin and chitosan in wound dressing applications. Biotechnol. Adv. 2011, 29, 322-337.

16. Mi, F.L.; Shyu, S.S.; Wu, Y.B.; Lee, S.T.; Shyong, J.Y.; Huang, R.N. Fabrication and characterization of a sponge-like asymmetric chitosan membrane as a wound dressing. Biomaterials 2001, 22, 165-173.

17. More, Y.; Willen, S.; Catalano, P. Management of early nasal polyposis using a steroid-impregnated nasal dressing. Int. Forum Allergy Rhinol. 2011, 1, 401-404.

18. Catalano, P.J.; Strouch, M. The minimally invasive sinus technique: Theory and practice. Otolaryn. Clin. N. Am. 2004, 37, 401-409.

(C) 2015 by the authors; licensee MDPI, Basel, Switzerland. This article is an open access article distributed under the terms and conditions of the Creative Commons Attribution license (http://creativecommons.org/licenses/by/4.0/). 